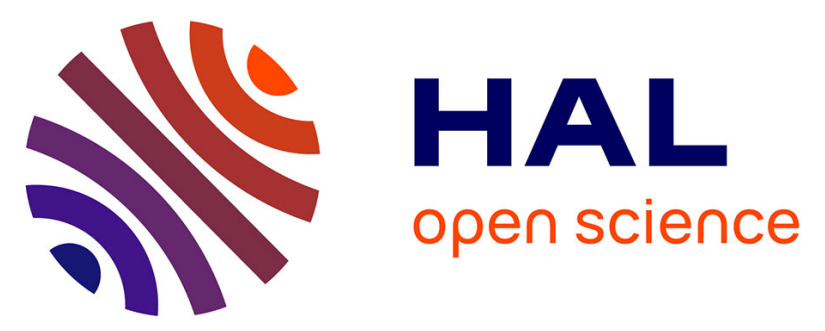

\title{
Ultra-flat, low-noise, and linearly polarized fiber supercontinuum source covering 670-1390 nm
}

Etienne Genier, Sacha Grelet, Rasmus D Engelsholm, Patrick Bowen, Peter Moselund, Ole Bang, John Michaël Dudley, Thibaut Sylvestre

\section{To cite this version:}

Etienne Genier, Sacha Grelet, Rasmus D Engelsholm, Patrick Bowen, Peter Moselund, et al.. Ultraflat, low-noise, and linearly polarized fiber supercontinuum source covering 670-1390 nm. Optics Letters, 2021, 46 (8), pp.1820-1823. hal-03403739

\section{HAL Id: hal-03403739 \\ https://hal.science/hal-03403739}

Submitted on 26 Oct 2021

HAL is a multi-disciplinary open access archive for the deposit and dissemination of scientific research documents, whether they are published or not. The documents may come from teaching and research institutions in France or abroad, or from public or private research centers.
L'archive ouverte pluridisciplinaire HAL, est destinée au dépôt et à la diffusion de documents scientifiques de niveau recherche, publiés ou non, émanant des établissements d'enseignement et de recherche français ou étrangers, des laboratoires publics ou privés. 


\section{An ultra-flat, low-noise and linearly polarized fiber supercontinuum source covering $670 \mathrm{~nm}-1390 \mathrm{~nm}$}

This paper was downloaded from TechRxiv (https://www.techrxiv.org).

LICENSE

CC BY 4.0

SUBMISSION DATE / POSTED DATE

10-03-2021 / 17-03-2021

\section{CITATION}

Genier, Etienne (2021): An ultra-flat, low-noise and linearly polarized fiber supercontinuum source covering 670 nm-1390 nm. TechRxiv. Preprint. https://doi.org/10.36227/techrxiv.14192114.v1

$\mathrm{DOI}$

10.36227/techrxiv.14192114.v1 


\title{
An ultra-flat, low-noise and linearly polarized fiber supercontinuum source covering 670 $\mathrm{nm}-1390 \mathrm{~nm}$
}

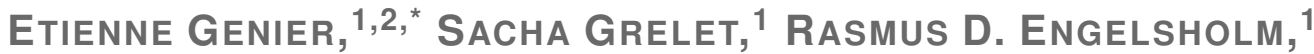 \\ Patrick Bowen, ${ }^{1}$ Peter M. Moselund, ${ }^{1}$ Ole Bang ${ }^{1,3}$ John M. \\ DudLey, ${ }^{2}$ AND ThIBAUt SYLVESTRE ${ }^{2}$ \\ ${ }^{1}$ NKT Photonics A/S, Blokken 84, DK-3460, Birkerød, Denmark \\ ${ }^{2}$ Institut FEMTO-ST, UMR 6174 CNRS-Université de Franche-Comté, 25030 Besançon, France \\ ${ }^{3}$ DTU Fotonik, Department of Photonics Engineering, Technical University of Denmark, $2800 \mathrm{Kgs}$. \\ Lyngby, Denmark \\ *etge@nktphotonics.com
}

\begin{abstract}
We report an octave-spanning coherent supercontinuum (SC) fiber laser with excellent noise and polarization properties. This was achieved by pumping a highly birefringent all-normal dispersion (ANDi) photonic crystal fiber with a compact high-power ytterbium femtosecond laser at $1049 \mathrm{~nm}$. This system generates an ultra-flat SC spectrum from $670 \mathrm{~nm}$ to $1390 \mathrm{~nm}$ with a power spectral density higher than $0.4 \mathrm{~mW} / \mathrm{nm}$ and a polarization extinction ratio of $17 \mathrm{~dB}$ across the entire bandwidth. An average pulse-to-pulse relative intensity noise (RIN) down to $0.54 \%$ from $700 \mathrm{~nm}$ to $1100 \mathrm{~nm}$ has been measured and found to be in good agreement with numerical simulations. This highly-stable broadband source could find strong potential applications in biomedical imaging and spectroscopy where improved signal to noise ratio is essential.
\end{abstract}

( 2021 Optical Society of America

Supercontinuum (SC) generation in all-normal dispersion (ANDi) photonic crystal fibers (PCF) has recently been investigated due to its ability to generate a low-noise broadband coherent spectrum [1-6], with a noise level much lower than typical SC sources using anomalous dispersion. The main issues with typical soliton-based SC generation is the poor flatness and the coherence degradation for a soliton number higher than 16, associated with large pulse-to-pulse fluctuations due to noise amplification by modulation instability (MI), soliton fission and rogue wave generation [7]. On the contrary, ANDi SC generation, which is essentially based on nonlinear coherent effects such as self-phase modulation (SPM) and subsequently optical wave breaking (OWB), has a high degree of coherence and pulse to pulse stability $[8,9]$. ANDi SC generation is thus a topic being extensively investigated for several applications such as optical coherent tomography [10], coherent amplification [11], nonlinear microscopy [12], hyperspectral stimulated Raman scattering microscopy [13] , and coherent anti-Stokes Raman scattering (CARS) spectroscopy [14]. Several recent studies have however shown some limitations on the input pulse duration due to stimulated Raman scattering (SRS) and polarization modulation instability (PMI) $[5,6,9,15,16]$. Specifically, it has been shown that for input pulse duration longer than $\sim 500 \mathrm{fs}$, SRS-induced noise amplification impacts on the SC pulse coherence and spectral flatness [9]. Furthermore, for input pulse duration longer than $\sim 120 \mathrm{fs}$, PMI can take place between the two orthogonal eigenmodes of the fiber, thus degrading the relative intensity noise (RIN) and the output polarization for non-polarization maintaining (PM) fibers [5].

In this work, we overcome these limitations by using a silica-based PM ANDi PCF pumped with a low-noise, short pulse duration (180 fs) compact ytterbium mode-locked laser at $1049 \mathrm{~nm}$. Using this system, symmetrically flat and ripple-free SC generation has been generated from $670 \mathrm{~nm}$ to $1390 \mathrm{~nm}$ with a maximum output power of $720 \mathrm{~mW}$. It is shown in particular that pumping on the fast axis of the fiber provides the flattest and most stable SC while removing the cross-phase modulation instability (XPMI) that has recently been evidenced in PM ANDi 
fibers [17]. A polarization extinction ratio (PER) of $17 \mathrm{~dB}$ and an average RIN of $0.54 \%$ from $700 \mathrm{~nm}$ to $1100 \mathrm{~nm}$ were further measured. We finally compare the spectrally-resolved RIN of our ANDi-fiber-based SC source with those recently published in the literature and we show excellent noise and polarization characteristics. The experimental setup used to observe and analyze coherent SC generation in the PM-ANDi PCF is shown in Fig. 1. As a pump laser, we used a femtosecond ytterbium solid-state mode-locked laser (Origami 10 HP, NKT Photonics) centered at $1049 \mathrm{~nm}$, delivering a $180 \mathrm{fs}$ pulse train at an $80 \mathrm{MHz}$ repetition rate with a maximum average power of $4 \mathrm{~W}$, a low RIN of $0.5 \%$, and a PER of $31 \mathrm{~dB}$. The laser output power was controlled using a variable neutral density (ND) filter. A half-wave plate was used to rotate the field polarization state at the fiber input. An aspheric lens with a focal length of $6 \mathrm{~mm}$ was used to couple the light into the fiber core. The fiber is a PM ANDi PCF from NKT Photonics (Model NL-1050-NEG-PM). This fiber has a core diameter of $2.4 \mu \mathrm{m}$, a relative hole diameter of $\mathrm{d} / \Lambda=$ 0.45 , a small hole-to-hole pitch of $\Lambda=1.44 \mu \mathrm{m}$, and a nonlinear coefficient of $\gamma=26.8 \mathrm{~W}^{-1} \mathrm{~km}^{-1}$ at $1040 \mathrm{~nm}$. The fiber's dispersion and loss profiles are shown as an inset in Fig. 1 [17]. The dispersion has an all-normal parabolic profile with a peak at $-13 \mathrm{ps} / \mathrm{nm} / \mathrm{km}$ at $1040 \mathrm{~nm}$. The PM property of the PCF is mainly stress-rod induced and it has a slightly elliptic core, which together gives it a high birefringence of $4.710^{-4}$ at the pump wavelength. The output spectrum is recorded using an optical spectrum analyser (OSA) and analysed as a function of input polarization and pump power, while the RIN was measured in another path using a monochromator as filter passband, two different fast photodetectors to cover the whole SC bandwidth and an oscilloscope.

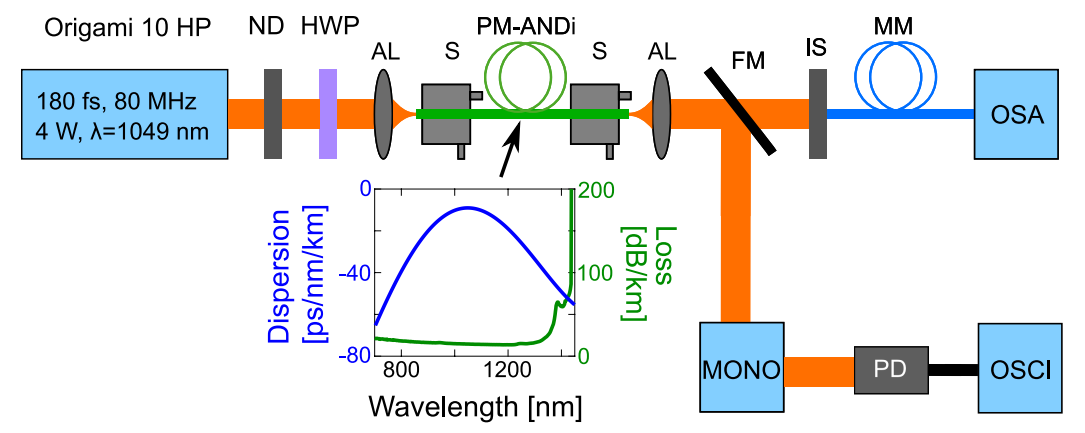

Fig. 1: Schematic of the setup, including ytterbium femtosecond mode-locked laser (ORIGAMI $10 \mathrm{HP}$ ), variable neutral density filter (ND), half wave plate (HWP), aspheric lenses (AL), 3D translation stages (S), $2 \mathrm{~m}$ of PM-ANDi PCF, flip-mirror (FM), integrating sphere (IS), $2 \mathrm{~m}$ of multimode pick-up fiber (MM), optical spectrum analyzer (OSA), monochromator (MONO), photodiode (PD), and oscilloscope (OSCI).

Figure 2 shows the spectral intensity profile for 3 selected polarizations and an average output power from the fiber of $720 \mathrm{~mW}$ (estimated input peak power of $48 \mathrm{~kW}$, including losses and Fresnel reflections). We obtain the broadest bandwidth while pumping along the slow axis (red curve), with a spectrum going from $685 \mathrm{~nm}$ to $1395 \mathrm{~nm}$ at $-3 \mathrm{~dB}$ if we disregard the oscillations in the central part of the spectrum. We find the spectrum becomes more noticeably modulated as we move closer to the center of the spectrum. Furthermore, the modulations appear together with the observation of an XPMI peak [17] when pumping along the slow axis. This clarifies the importance of avoiding XPMI, since the modulation can be detrimental to interference based imaging. The spectrum is the narrowest when pumping at $45^{\circ}$ from the principal axes (pink curve) spanning from $700 \mathrm{~nm}$ to $1370 \mathrm{~nm}$ at $-3 \mathrm{~dB}$, which is expected because only half of the power is available on each axis. We observe that the spectrum for $45^{\circ}$ is highly modulated from $870 \mathrm{~nm}$ to $1120 \mathrm{~nm}$. The XPMI sideband is again present and slightly stronger than the one obtained on the red curve, as expected from the theory [17-19]. Then, we obtain an extremely 
flat spectrum when pumping on the fast axis (blue curve) with a spectrum spanning from $670 \mathrm{~nm}$ to $1390 \mathrm{~nm}$ at $-3 \mathrm{~dB}$ without any trace of an XPMI sideband around $1400 \mathrm{~nm}$.

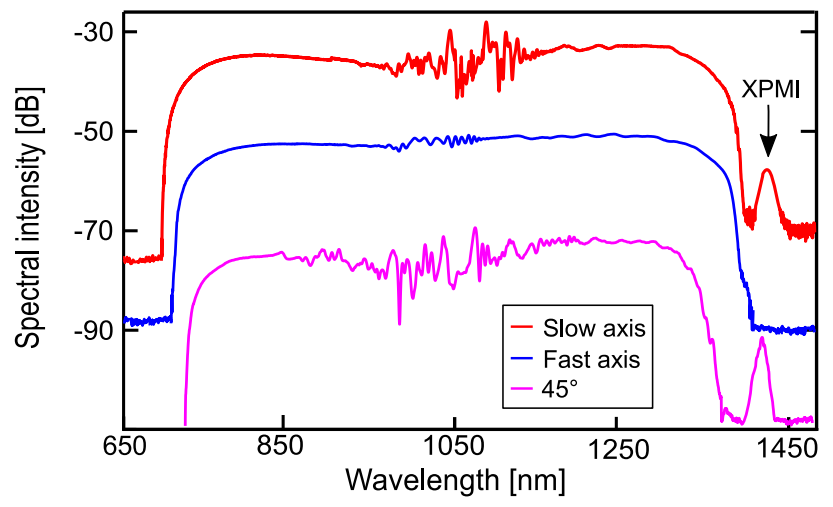

Fig. 2: Experimental SC spectra at the output of the PM-ANDi PCF for output average power of $720 \mathrm{~mW}$ with an input pulse duration of $180 \mathrm{fs}$ while pumping along the slow axis (red curve), fast axis (blue curve) and at $45^{\circ}$ from the axes (pink curve). The blue and red curves are offset by $15 \mathrm{~dB}$ and $30 \mathrm{~dB}$ from the pink curve, respectively.

We now investigate the case with pumping along the fast axis, for which we observed the best spectrum with the least ripples. Thus, Fig. 3 shows the evolution of the SC generation for a fiber output average power increasing from $1 \mathrm{~mW}$ to $720 \mathrm{~mW}$ (peak power going from $8 \mathrm{~W}$ to 48 $\mathrm{kW}$ ). We observe two SPM fringes on the pink curve (for a power of $6 \mathrm{~mW}$ ). Then, by increasing further the power up to $30 \mathrm{~mW}$ (brown spectrum), we can clearly see the appearance of OWB on the edges. Furthermore, the SC bandwidth goes from $9 \mathrm{~nm}$ (in the linear regime) to $720 \mathrm{~nm}$ (going from $670 \mathrm{~nm}$ to $1390 \mathrm{~nm}$ ) at $-3 \mathrm{~dB}$, which is more than one octave with a power spectral density (PSD) higher than $0.4 \mathrm{~mW} / \mathrm{nm}(-4 \mathrm{dBm} / \mathrm{nm})$. Next, as expected from coherent ANDi SC generation, the spectrum broadens symmetrically and is extremely flat (the maximum amplitude of the ripples in the SPM area is $1.65 \mathrm{~dB}$ ). Finally, we do not observe any SRS components in the final spectra at high power (red and black spectra) across the whole bandwidth [5,9]. Thus, we can expect this SC generation to be extremely low-noise and highly stable.

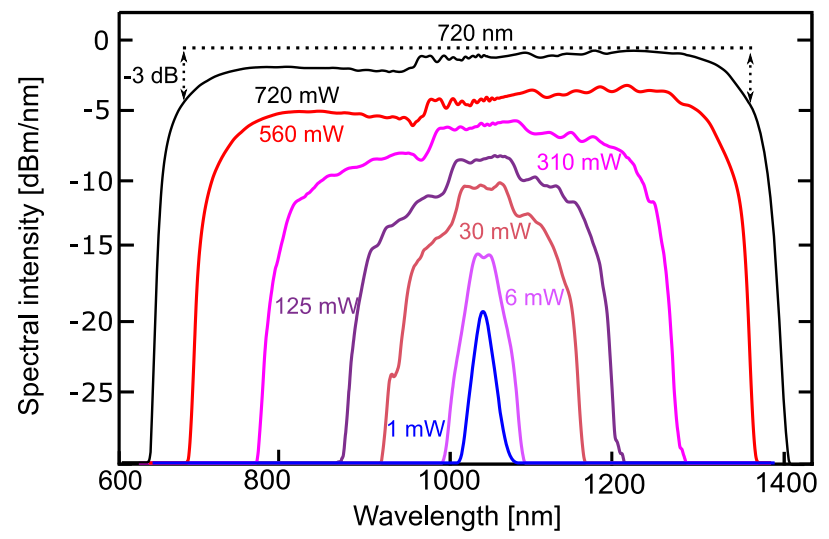

Fig. 3: Experimental SC spectra at the output of the PM-ANDi PCF for output average power of $1 \mathrm{~mW}$ to $720 \mathrm{~mW}$ with an input pulse duration of $180 \mathrm{fs}$ and an input beam polarized along the fast axis. 
Using a polarizer at the output of the ANDi-PCF, we measured the PER of the generated SC using the OSA. Fig. 4 depicts the SC spectra when the input beam is polarized along the fast axis and the output polarizer is aligned either on the fast axis (red curve) or on the slow axis (blue curve). Specifically, the polarizer is rotated to measure the minimum power including the whole spectrum and then the spectrum is measured at this angle and at a 90 degree rotation. This way, we can measure an average PER of $17 \mathrm{~dB}$ over the full SC bandwidth, which means that the SC output is nearly linearly polarized. We noticed however a PER degradation of $14 \mathrm{~dB}$ compared to the input pump laser (PER of $31 \mathrm{~dB}$ ). This degradation of $14 \mathrm{~dB}$ is also measured at low power (no spectral broadening) and may be explained by the collapse of the air holes at the entrance of the fiber required to remove back-reflection in the laser cavity.

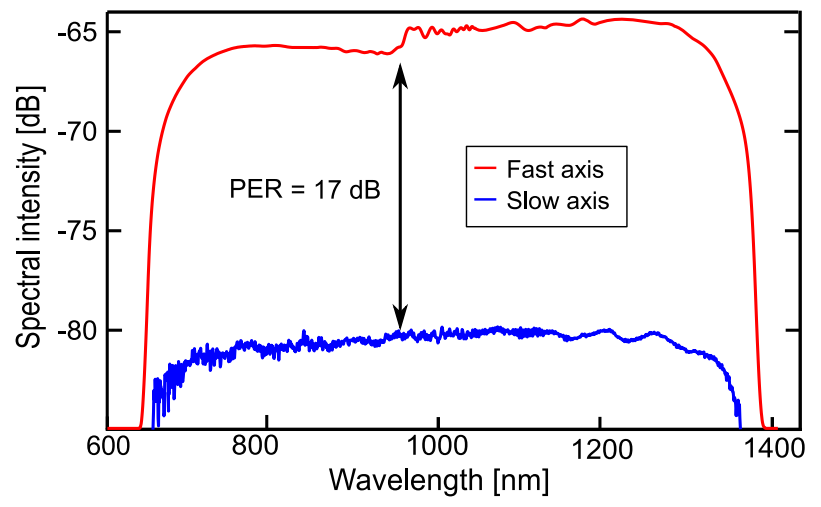

Fig. 4: Experimental SC spectra when pumping on the fiber's fast axis and using a polarizer aligned either on the fast axis (red) or on the slow axis (blue). The difference between the flat parts of the spectra gives a PER of about $17 \mathrm{~dB}$.

Let us now turn our attention to the noise properties of the pump and the generated supercontinuum. For the pump noise measurement, we coupled the output beam onto a silicon photodiode connected to a fast oscilloscope (DSA91204A from Agilent). After recording 10000 pulses, we obtained the histogram depicted in Fig. 5. Using a Gaussian fit of this distribution, we extract a mean value of 2.5 and a standard deviation of 0.011 . Thus, calculating the ratio of the standard deviation to the mean, we obtain a RIN value of $0.44 \%$ using this method, in rather good agreement with the laser manufacturer data $(0.5 \%)$.

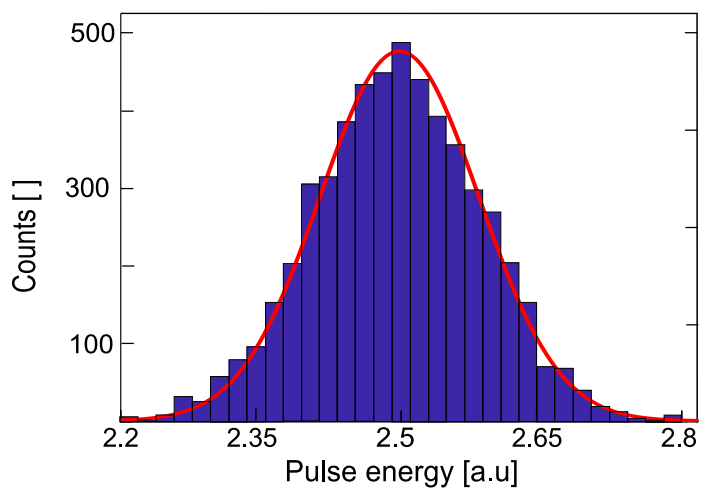

Fig. 5: Histogram of the variation of the energy of 10000 pulses measured with the oscilloscope. The red curve represents a Gaussian fit of the distribution. 
After measuring the pump laser noise, we further investigated the RIN over the whole SC bandwidth. To this end, we used a monochromator (from Princeton instrument) as a tunable spectral filter and the spectrally-sliced SC was sent to the photodetector and oscilloscope. We specifically used two photodetectors to cover the SC bandwidth: a silicon photodetector from 650 to $1100 \mathrm{~nm}$ and an InGaAs photodetector for longer wavelengths. The SC RIN was still measured using the Gaussian fit method by saving 10000 pulses onto an oscilloscope (PicoScope) every $10 \mathrm{~nm}$, with a $3 \mathrm{~nm}$ bandwidth for the silicon photodetector and a $1 \mathrm{~nm}$ bandwidth for the InGaAs one. Fig. 6 depicts the two spectrally-resolved RIN measurements as red dots (silicon) and as green dots (InGaAs), while the blue curve shows the related SC spectrum while pumping the fiber at $45^{\circ}$ off the axes. We find an average RIN from $700 \mathrm{~nm}$ to $1100 \mathrm{~nm}$ down to $0.54 \%$, when we omit the high edge RIN values. The results also show that the RIN increases drastically on the SC edges due to both the reduction of the SC power and the strong effect of laser peak power fluctuations on the supercontinuum bandwidth [6]. Furthermore, the pink curve in Fig. 6 depicts the estimated minimum RIN values measured by our system as a function of wavelength. Indeed, our scope has an estimated dark noise standard deviation of $1 \mathrm{mV}$. Thus, if we divide this number by the mean of our Gaussian distribution, for each spectrally resolved bin, we obtain a minimum estimated spectrally resolved RIN value of $0.14 \%$. Finally, we observe that our measured spectrally RIN values are all slighty above the estimated minimum.

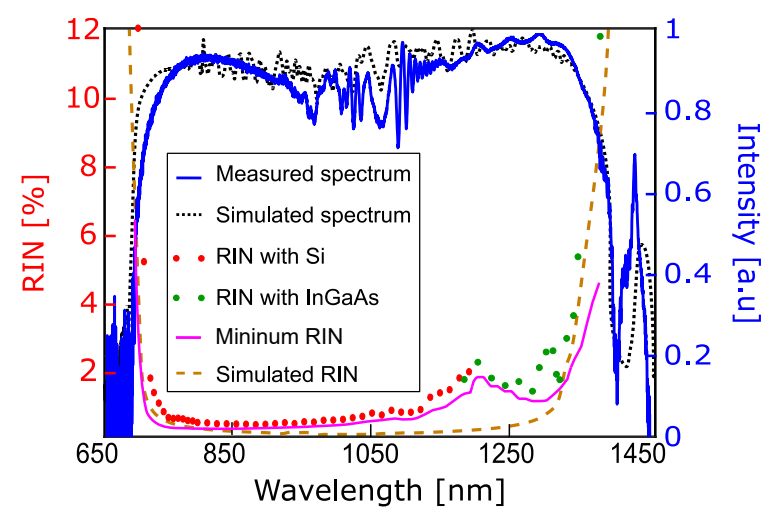

Fig. 6: Measured spectrally-resolved RIN using the oscilloscope method with silicon (red dots) and InGaAs (green dots) photodetector and corresponding average normalized SC spectrum (solid blue), pumping at $45^{\circ}$ from the principal axes plus simulated SC (black dots) and RIN spectra (dashed brown). The pink curve shows the estimated minimum RIN values from our measurement system.

These experimental measurements have been further compared to numerical simulations of the two-coupled generalized nonlinear Schrödinger equations (CGNLSEs), as those described in Ref. [20] for highly-birefringent PM fibers. We performed SC and RIN simulations solving the CGNLSEs using the split-step method and the initial conditions as described in [6] with an input pulse duration of $180 \mathrm{fs}$ (full width half maximum), an input peak power of $48 \mathrm{~kW}$, an input amplitude noise value of $0.22 \%$ (amplitude noise $\approx \mathrm{RIN} / 2$ ) and associated anti-correlated pulse duration fluctuations to keep the energy of the mode-locked laser constant. To plot the simulated RIN, we used the following equation with an ensemble size of 50 independent pulses, which is sufficient to minimise modelling error:

$$
\operatorname{RIN}(\lambda)=\sqrt{\left\langle\left(|\tilde{A}(\lambda)|^{2}-\left\langle|\tilde{A}(\lambda)|^{2}\right\rangle\right)^{2}\right\rangle} /\left\langle|\tilde{A}(\lambda)|^{2}\right\rangle .
$$


The simulation results are shown in Fig. 6 (black dots for the spectrum and dashed brown for the RIN). As can be seen, the agreement is excellent with the experimental spectrum, both for the shape and bandwidth, with a highly modulated spectrum and the generation of an XPMI sideband near $1400 \mathrm{~nm}$. This agreement is also rather good between the measured and simulated RIN.

\begin{tabular}{|c|c|c|c|c|c|}
\hline Ref & $\Delta \lambda_{S C}$ & Method & $\Delta \lambda_{R I N}$ & $\Delta \lambda_{\text {filter }}$ & RIN \\
\hline$[5]$ & $620 \mathrm{~nm},-10 \mathrm{~dB}$ & Osci. & $600 \mathrm{~nm}$ & $10 \mathrm{~nm}$ & $33 \%$ \\
\hline$[21]$ & $480 \mathrm{~nm},-30 \mathrm{~dB}$ & Osci. & $200 \mathrm{~nm}$ & $12 \mathrm{~nm}$ & $1.2 \%$ \\
\hline$[11]$ & $300 \mathrm{~nm},-3 \mathrm{~dB}$, & ESA & $300 \mathrm{~nm}$ & & $0.07 \%$ \\
\hline Ours & $720 \mathrm{~nm},-3 \mathrm{~dB}$ & Osci. & $400 \mathrm{~nm}$ & $3 \mathrm{~nm}$ & $0.54 \%$ \\
\hline
\end{tabular}

Table 1: Average RIN values in ANDi SC generation from the current literature and our system.

Table 1 compares the noise performances of our SC source to those of the literature [5,21]. The second column indicates the SC bandwidth. The third one explained the method used to measure the RIN of the SC system, either based on an oscilloscope in the time domain or on an Electric spectrum analyser (ESA) in the frequency domain. The fourth and fifth ones stipulate the measurement and filter bandwidth to obtain the average RIN specified in the last column. Few methods have indeed been implemented to measure the coherence or the RIN of ANDi SC generation [5, 21, 22]. For the coherence, in [22], the authors used a dispersive Fourier transform technique to highlight the stability of ANDi SC generation. Then, using a fiber-based unequal-path Michelson interferometer, they demonstrate a high fringe visibility meaning a high degree of coherence for the ANDi SC generation. Also, in Refs. [5] and [21], the authors used a setup composed of bandpass filters $(10 / 12 \mathrm{~nm})$, a fast photodiode and an oscilloscope to measure the spectrally-resolved RIN of the SC spectrum. In [5], the authors reported an average RIN of $33 \%$. This value can be considered high but, in this study, the coherence of ANDi SC generation was degraded by the PMI effect. In [21], the authors reported a very low average RIN of $1.2 \%$, due to the low input peak power used to obtain their ANDI SC and the large bandwidth of their filters.

From Table 1, we see that we obtain the lowest average RIN value using the oscilloscope measurement method. About the value reported by [11], it is not possible to compare directly our RIN value to the $0.07 \%$ announced because they don't use any passband filters and thus measure a single RIN value for the whole SC spectrum. Furthermore, they limit their ESA measurement bandwidth from $10 \mathrm{~Hz}$ to $20 \mathrm{MHz}$ while the repetition rate of their laser is up to 80 MHz. Therefore, it reduces the overall RIN value. Finally, we underline the setup composed by the Origami 10 HP laser and the PM-ANDi silica PCF is the system with the lowest average RIN to our knowledge and the highest PER (measured PER of $10 \mathrm{~dB}$ in [11]), and thus it can be also considered as one of the state-of-the-art systems.

In conclusion, using a compact ytterbium mode-locked laser producing $180 \mathrm{fs}$ pulses and a highly-nonlinear polarization-maintaining ANDi PCF, we were able to generate an ultra-flat octave-spanning SC spectrum with a bandwidth up to $720 \mathrm{~nm}$ (from $670 \mathrm{~nm}$ to $1390 \mathrm{~nm}$ ) and associated PSD higher than $0.4 \mathrm{~mW} / \mathrm{nm}$. We have shown that the SC is linearly polarized with a PER of $17 \mathrm{~dB}$ across the whole spectrum bandwidth and with a spectrally-resolved RIN below $0.54 \%$ from $700 \mathrm{~nm}$ to $1100 \mathrm{~nm}$, which is, to the best of our knowledge, the lowest average SC ANDi RIN value ever reported with this measurement method. This system could find many applications in OCT, metrology, or as a wavelength-tunable fs laser source thanks to its octave-spanning bandwidth, flatness, low-noise, high coherence, polarization and near-linear chirp properties. 
Funding. Horizon 2020 Framework Programme (722380); Agence Nationale de la Recherche (ANR) (ANR-15-IDEX-0003, ANR-17-EURE-0002).

Disclosures. The authors declare no conflicts of interest.

\section{References}

1. T. Hori, J. Takayanagi, N. Nishizawa, and T. Goto, "Flatly broadened, wideband and low noise supercontinuum generation in highly nonlinear hybrid fiber,” Opt. Express 12, 317-324 (2004).

2. L. E. Hooper, P. J. Mosley, A. C. Muir, W. J. Wadsworth, and J. C. Knight, "Coherent supercontinuum generation in photonic crystal fiber with all-normal group velocity dispersion,” Opt. Express 19, 4902-4907 (2011).

3. K. Tarnowski, T. Martynkien, P. Mergo, K. Poturaj, A. Anuszkiewicz, P. Béjot, F. Billard, O. Faucher, B. Kibler, and W. Urbanczyk, "Polarized all-normal dispersion supercontinuum reaching $2.5 \mu \mathrm{m}$ generated in a birefringent microstructured silica fiber," Opt. Express 25, 27452-27463 (2017).

4. A. Hartung, A. M. Heidt, and H. Bartelt, "Design of all-normal dispersion microstructured optical fibers for pulse-preserving supercontinuum generation," Opt. Express 19, 7742-7749 (2011).

5. I. B. Gonzalo, R. D. Engelsholm, M. P. Sørensen, and O. Bang, "Polarization noise places severe constraints on coherence of all-normal dispersion femtosecond supercontinuum generation," Sci. Reports 8, 6579 (2018).

6. E. Genier, P. Bowen, T. Sylvestre, J. M. Dudley, P. Moselund, and O. Bang, "Amplitude noise and coherence degradation of femtosecond supercontinuum generation in all-normal-dispersion fibers," J. Opt. Soc. Am. B 36, A161-A167 (2019).

7. J. M. Dudley, G. Genty, and S. Coen, "Supercontinuum generation in photonic crystal fiber," Rev. Mod. Phys. 78 1135 (2006).

8. C. Finot, B. Kibler, L. Provost, and S. Wabnitz, "Beneficial impact of wave-breaking for coherent continuum formation in normally dispersive nonlinear fibers," J. Opt. Soc. Am. B 25, 1938-1948 (2008).

9. A. M. Heidt, J. S. Feehan, J. H. V. Price, and T. Feurer, "Limits of coherent supercontinuum generation in normal dispersion fibers," J. Opt. Soc. Am. B 34, 764-775 (2017).

10. M. Jensen, I. B. Gonzalo, R. D. Engelsholm, M. Maria, N. M. Israelsen, A. Podoleanu, and O. Bang, "Noise of supercontinuum sources in spectral domain optical coherence tomography," J. Opt. Soc. Am. B 36, A154-A160 (2019).

11. A. M. Heidt, J. M. Hodasi, A. Rampur, D.-M. Spangenberg, M. Ryser, M. Klimczak, and T. Feurer, "Low noise all-fiber amplification of a coherent supercontinuum at $2 \mu \mathrm{m}$ and its limits imposed by polarization noise," Sci. Reports 10, 1-9 (2020).

12. G. Dwapanyin, D. Spangenberg, A. Heidt, T. Feurer, G. Bosman, P. Neethling, and E. Rohwer, "Generalized spectral phase-only time-domain ptychographic phase reconstruction applied in nonlinear microscopy," J. Opt. Soc. Am. B 37, A285-A292 (2020).

13. P. Abdolghader, A. F. Pegoraro, N. Y. Joly, A. Ridsdale, R. Lausten, F. Légaré, and A. Stolow, "All normal dispersion nonlinear fibre supercontinuum source characterization and application in hyperspectral stimulated raman scattering microscopy,” Opt. Express 28, 35997-36008 (2020).

14. R. Viljoen, P. Neethling, D. Spangenberg, A. Heidt, H.-M. Frey, T. Feurer, and E. Rohwer, "Implementation of temporal ptychography algorithm, i 2 pie, for improved single-beam coherent anti-stokes raman scattering measurements," J. Opt. Soc. Am. B 37, A259-A265 (2020).

15. U. Møller and O. Bang, "Intensity noise in normal-pumped picosecond supercontinuum generation, where higher-order raman lines cross into anomalous dispersion regime," Electron. Lett. 49, 63-65 (2013).

16. J. S. Feehan and J. H. Price, "Decoherence due to xpm-assisted raman amplification for polarization or wavelength offset pulses in all-normal dispersion supercontinuum generation,” J. Opt. Soc. Am. B 37, 635-644 (2020).

17. E. Genier, A. N. Ghosh, S. Bobba, P. Bowen, P. M. Moselund, O. Bang, J. M. Dudley, and T. Sylvestre, "Cross-phase modulation instability in pm andi fiber-based supercontinuum generation," Opt. Lett. 45, 3545-3548 (2020).

18. A. Kudlinski, A. Bendahmane, D. Labat, S. Virally, R. Murray, E. Kelleher, and A. Mussot, "Simultaneous scalar and cross-phase modulation instabilities in highly birefringent photonic crystal fiber," Opt. Express 21, 8437-8443 (2013).

19. P.D. Drummond, T.A.B. Kennedy, J.M. Dudley, R. Leonhardt, and J.D. Harvey, "Cross-phase modulational instability in high-birefringence fibers," Opt. Commun. 78, 137-142 (1990).

20. A. N. Ghosh, M. Meneghetti, C. R. Petersen, O. Bang, L. Brilland, S. Venck, J. Troles, J. M. Dudley, and T. Sylvestre, "Chalcogenide-glass polarization-maintaining photonic crystal fiber for mid-infrared supercontinuum generation," J. Physics: Photonics 1, 044003 (2019).

21. S. Rao D.S., R. D. Engelsholm, I. B. Gonzalo, B. Zhou, P. Bowen, P. M. Moselund, O. Bang, and M. Bache, "Ultra-low-noise supercontinuum generation with a flat near-zero normal dispersion fiber," Opt. Lett. 44, 2216-2219 (2019).

22. K. Tarnowski, T. Martynkien, P. Mergo, J. Sotor, and G. Soboń, "Compact all-fiber source of coherent linearly polarized octave-spanning supercontinuum based on normal dispersion silica fiber," Sci. Reports 9, 1-8 (2019). 